http://dx.doi.org/10.18778/2196-8403.2015.02

IN MEMORIAM

Martin Grimberg

* 1959 - † 2015

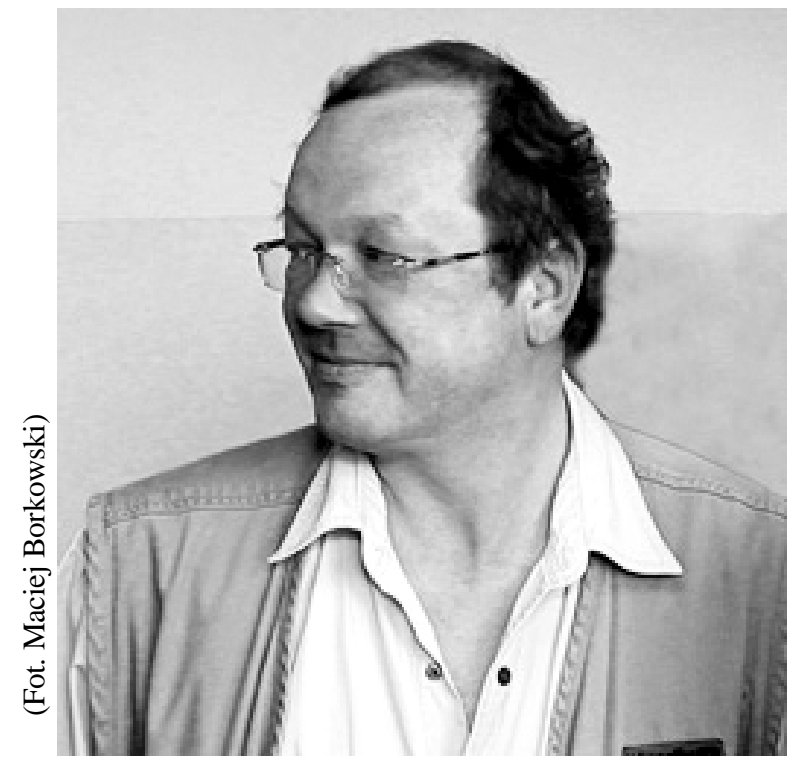





\section{Martin Grimberg. Ein Nachruf}

Am 2. Juli 2015 verstarb nach schwerer Krankheit Dr. Martin Grimberg, Mitbegründer, langjähriger Redaktionsleiter und Mitherausgeber von CoNVIVIUM, dem vom DAAD initiierten Germanistischen Jahrbuch Polen. Geboren 1959, aufgewachsen im Ruhrgebiet, studierte und promovierte er ,magna cum laude bei Siegfried Grosse in Bochum mit Untersuchungen zum Verlust der Schriftsprachlichkeit. Im Mittelpunkt stand die Frage nach der Entstehung literaler Alltagskommunikation im Zuge der allgemeinen Schulpflicht, ihrer Eindämmung durch behördliche Vorgaben wie die Stiehlschen Regulative von 1854, die zu große Unterschichtenbildung in Preußen verhindern sollten, und schließlich ihrem Rückgang im Zuge der Ablösung klassisch-skripturaler Räume während des 20. Jahrhunderts. Auch in anderen frühen Arbeiten widmete er sich Alltagstexten (insbesondere Briefen) literaturferner Bevölkerungsgruppen vorrangig im Kaiserreich. Früh schon fällt sein Interesse für Polen und für deutsch-polnische Interferenzen in den untersuchten Sprachzeugnissen auf. Während der 1980er Jahre hatte er für die Robert-Bosch-Stiftung Deutsch in Polen unterrichtet, genauer: in Posen, das ihm zweite Heimat werden sollte, die Druckausgabe seiner Dissertation hatte er seinen polnischen Freunden gewidmet.

Die intensive Beschäftigung mit der Schreibsprache wurde in praktischer Hinsicht zentrales Rückgrat seiner späteren Redaktionstätigkeit. Erste Redaktionserfahrung erwarb er, damals aktiver Sportler, ebenfalls noch während der 1980er Jahre als Leiter der von ihm systematisch professionalisierten Sportzeitschrift Der Wurfger. Nach der politischen Wende in Ostmitteleuropa gehörte er zur ersten Generation vom DAAD nach Polen entsandter Lektoren. Da das DAAD-Lektorat in Posen bereits vergeben war, ging er als DAAD-Lektor nach Oppeln in Oberschlesien. Die Germanistik dort war erst nach der politischen Wende entstanden - da es in der Region eine bis dahin nicht anerkannte deutsche Minderheit gab, hatte das kommunistische System in der Region keine Germanistik zugelassen.

Als Grimberg nach Oppeln kam, befand sich das Institut noch in der Gründungsphase. Bis 1994 wurde es auf zweiter Stelle von Grażyna Barbara Szewczyk von Katowice aus geleitet, die übrigen Mitarbeiter arbeiteten teilweise selbst nur auf zweiter Stelle in Oppeln, teils waren sie eben erst mit dem Magister-Studium fertig geworden. Viele verdienten beträchtliche Teile ihres Lebensunterhalts unter den damals sehr schwierigen Umständen mit anderen Tätigkeiten. Grimberg, solchen Zwängen nicht unterworfen, konnte sich als 
Einziger voll in den Institutsaufbau einbringen und sicherte so noch über seine Zeit hinaus dem DAAD-Lektor eine in dieser Form einmalige zentrale Position am Institut in Oppeln.

Hinzu kam ein weiteres Problem: Die damals heikle politische Situation mit einem schwelenden, zum Glück langsam eingeschlafenen Nationalitätenkonflikt in der Region und widersprüchliche Erwartungen verschiedener kultureller und politischer Gruppierungen aus Polen und Deutschland an Institut und DAAD-Lektor mussten ausbalanciert werden. Grimberg bewies dabei großes Geschick. Er ließ sich nicht vereinnahmen (legendär war seine Fähigkeit zu schweigen und Erwartungen an ihn ins Leere laufen zu lassen) und war beteiligt an Lösungen, die jeder nationalen Frontziehung zuwiderliefen. So gab es von deutscher Seite aus Überlegungen, aufgrund der langen politischen Benachteiligung der deutschen Minderheit und ihrer oft geringen Deutschkenntnisse spezielle Stipendien nur für Angehörige derselben unter den GermanistikStudenten in Oppeln einzuführen - was wiederum fast zwangsläufig zu ungleicher Chancenverteilung und vielleicht sogar Spannungen unter den Studierenden (wohl auch über die Universität hinaus) hätte führen müssen. Unter maßgeblicher Beteiligung Grimbergs wurde das - mittlerweile längst überflüssige und ausgelaufene - Stipendienprogramm schließlich ausgeschrieben für alle Germanistik-Studierenden aus der Region, ungeachtet ihrer nationalen Selbstzuordnung. Bei der Vergabe wurde bewusst nicht darauf geachtet, welcher Nation sich ein Bewerber zuschrieb. Die Betreuung dieses Programms lag Grimberg besonders am Herzen, er muss über die Jahre Hunderte Sprachzeugnisse und Gutachten ausgestellt und verfasst haben.

Der penible Arbeiter, der Grimberg immer war, zeigte sich in Oppeln an vielen Dingen - nicht zuletzt an dem von ihm aufgebauten DAAD-Handapparat mit mehreren tausend Büchern. Als weltweit größter DAAD-Handapparat bildete er zugleich das Kernstück der Institutsbibliothek; da deren Bücher großenteils offiziell dem DAAD gehörten, war diese vor dem steten Verlangen der Hauptbibliothek nach ihren Beständen geschützt. Ursache dieses Begehrens waren - politischen Gründen geschuldete - bibliothekarische Großspenden (etwa des Landes Nordrhein-Westfalen) an die Oppelner Germanistik, an deren Zustandekommen auch Martin Grimberg beteiligt war. Er konnte in großen Dimensionen denken und organisieren und sich zugleich im bürokratischen KleinKlein umsichtig durchsetzen.

In diesen Jahren war er außerdem im Umfeld von Ulrich Engels Großprojekt der deutsch-polnischen Grammatik hilfreich tätig: Für die von Engel und Eugeniusz Tomiczek regelmäßig veranstalteten Projekttreffen in Karpacz 
übernahm er als DAAD-Lektor die Rolle des Mitveranstalters und Antragsstellers, was ihm enge Kontakte zu vielen führenden polnischen Linguisten einbrachte.

Doch damit nicht genug: Während seiner Zeit als DAAD-Lektor in Oppeln beschloss der DAAD, die einst von Lektoren der DDR herausgebrachten germanistischen Jahrbücher für die verschiedenen Länder Ostmitteleuropas in neuer Form wiederzubeleben. Für Polen bildete sich ein Redaktionskomitee aus Barbara Breysach, Michael Ewert, Martin Grimberg, Gabriel Falkenberg, Ursula Kiermaier, Brigitta Niehaus, Frauke Peters und Birgit Rek, das das erste Jahrbuch gemeinsam redigieren und herausgeben sollte. Nachdem der erste Koordinator Falkenberg hatte ausscheiden müssen, übernahm Grimberg dessen Rolle als (so steht es im Impressum) „Koordinator und verantwortlicher Herausgeber für diesen ersten Band“ des, wie es damals hieß, Deutsch-polnischen Jahrbuchs der Germanistik, das 1993 erschien. Der zweite Band von 1994 hieß dann bereits ConvIVIUM, die Redaktionsmitglieder (zu denen noch Frank König gestoßen war) firmierten abermals als Herausgeber, „Koordinator und verantwortlicher Herausgeber für diesen Band“ war wieder Martin Grimberg. Er verdankte diese Sonderrolle seiner peniblen redaktionellen Tätigkeit, die auch die oft quälend kleine Detailarbeit nicht scheute, was ihn automatisch in den Mittelpunkt aller Arbeitsabläufe stellte. Dass es in der Redaktion dennoch Diskussionen weniger um die Arbeit (um die kümmerte sich Grimberg) als um symbolische Posten wie die Herausgeberschaft gab, zeigt sich am sukzessiv sich verändernden Impressum: 1995 gab es „Redaktion und Herausgeber“ einerseits, „Verantwortliche Herausgeber“ anderseits. Letztere waren nun und fortan (in dieser Reihenfolge) Martin Grimberg, Ulrich Engel und Stefan H. Kaszyński. Engel und Kaszyński waren vom DAAD und Wissenschaftlichem Beirat für diese Rolle nominiert worden, um dem Jahrbuch und dem Hauptträger der Arbeit an ihm mehr Stabilität zu verleihen. Mit dem Jahrbuch 1996 hatte sich die bis heute gültige Struktur von ConvIVIUM herausgebildet - mit der jährlichen gemeinsamen Sitzung von Beirat und Redaktion als eigentlichem Entscheidungsgremium, dem Redaktionsleiter Grimberg als erstgenanntem Herausgeber und zwei Mitherausgebern, von denen einer das Fach Linguistik repräsentiert, der andere die Literaturwissenschaft.

ConviviUm hatte sich aber auch nach außen hin durchzusetzen im Konkurrenzkampf mit den zahlreichen germanistischen und philologischen Zeitschriften Polens. Dies gelang durch den international zusammengesetzten Beirat mit namhaften Wissenschaftlern aus Polen und Deutschland, die sich einmal jähr- 
lich mit der Redaktion treffen. Diese Treffen sind das eigentliche Entscheidungs- und Machtzentrum von CoNviviUM, alle wesentlichen Beschlüsse werden von ihnen gefasst, die Inhaltsverzeichnisse der einzelnen Ausgaben durch sie diskutiert und beschlossen. Von Anfang an setzte Grimberg auf ein peerreview- und double-blind-Verfahren bei der Begutachtung von Beiträgen, von den dafür zuständigen Beiratsmitgliedern verlangte er detaillierte, jede Kritik ausführlich begründende Gutachten. Diese wurden und werden von vielen polnischen Nachwuchswissenschaftlern zugleich gefürchtet und dankbar entgegengenommen. Die auf Anmahnen Grimbergs (fast) immer in unterstützendem Ton vorgetragene Kritik ist in der Sache stets hart, in vielen Fällen aber lehrund hilfreich. Dass gelegentlich auch ein zu schnell geschriebener Text einer anerkannten Fachgröße berechtigte Kritik erhielt, war dem Ruf von CoNVIVIUM durchaus zuträglich - Grimberg war nie ein Freund der Rücksichtnahme auf Name, Position oder auch persönliche Bekanntschaft; selbst Mitglieder des Wissenschaftlichen Beirats mussten die Ablehnung von ihnen eingereichter Artikel hinnehmen.

1996 endete das DAAD-Lektorat von Martin Grimberg und damit auch seine Tätigkeit als Redaktionsleiter und Mit- und Hauptherausgeber von CONVIVIUM. Er konnte mir als seinem Nachfolger ein bereits hervorragend aufgestelltes und entwicklungsfähiges Organ übergeben. Grimberg ging zunächst nach Deutschland zurück, doch zog es ihn bald wieder nach Polen. 1998 nutzte er die Gelegenheit, um das DAAD-Lektorat in Warschau zu übernehmen. Als 2002 das DAAD-Lektorat in Posen frei wurde, konnte er sich endlich seinen alten Traum erfüllen und an die Adam-Mickiewicz-Universität in Posen wechseln, wo er sich bald auch häuslich und endgültig einrichtete. 2002 übernahm er auch wieder die Rolle des Mitveranstalters der Karpacz-Tagungen, die sich in der Zwischenzeit von reinen Projekttagungen zu wissenschaftlichen Nachwuchstagungen gewandelt hatten. In den in diesem Rahmen entstandenen Tagungsbänden wird er als Mitherausgeber genannt - bis 2005 (2006 hörte der DAAD auf, diese Tagungen finanziell zu fördern).

Im selben Jahr 2002 übernahm Grimberg auch wieder CoNVIVIUM. 2001 war ich nach dem Ende meines DAAD-Lektorats nach Deutschland zurückgekehrt. Grimberg war aufgrund seiner engen Kenntnis des Jahrbuchs und der technischen Abläufe der geeignetste Nachfolgekandidat und so stimmte der DAAD zu, dass er Convivium wieder leiten solle. Das Jahrbuch wurde nun sein eigentliches Haupt- und Lebenswerk. Mit derselben Leidenschaft, mit der er es aufgebaut hatte, führte er es fort. Dies gilt insbesondere auch für die noch zu meiner Zeit im Rahmen von ConviviUM etablierte Jahresbibliographie der 
polnischen Germanistik, die von 2002 an aufgrund ihres wachsenden Umfangs als Beiheft zu Convivium im Zweijahresabstand erschien. Daraus entwickelte sich ein fast schon enzyklopädisch anmutendes bibliographisch-detektivisches Großunternehmen, das Grimberg mit enormem Arbeitseinsatz weiter vorantrieb. Hätte er nicht eigenhändig all die vielen Titel eingegeben und überprüft, hätte die Bibliographie trotz umfangreicher Unterstützung (etwa durch Maria Sawicka, Lesław Cirko oder Gabriela Ociepa) nie so lange erscheinen können - der bisher letzte Band war 2011 für die Jahre 2008 und 2009 erschienen, die Arbeit an der Ausgabe 2013 für die Jahre 2010 und 2011 hat Grimberg trotz des Kampfes mit seiner Krankheit weit vorangetrieben. Ob freilich jemand die Zeit und die Kraft finden wird, diese selbstlose Arbeit fortzusetzen, ist im Moment nicht abzusehen.

Wie auch immer: Ohne Martin Grimberg wäre ConvIVIUM nie geworden, was es geworden ist.

Jürgen Joachimsthaler für den wissenschaftlichen Beirat 